\title{
Factors of importance for decreased early embryo survival in female mice treated neonatally with diethylstilboestrol
}

\author{
A. Halling ${ }^{1}$, C. von Mecklenburg ${ }^{2}$ and J-G. Forsberg ${ }^{1}$ \\ ${ }^{1}$ Department of Anatomy, University of Lund, Biskopsgatan 7, S-223 62 Lund, Sweden; and \\ ${ }^{2}$ Department of Oncology and Electron Microscopy Unit, University of Lund, Lund University \\ Hospital, S-221 85 Lund, Sweden
}

\begin{abstract}
Neonatal female mice of the NMRI strain were treated s.c. with $5 \mu \mathrm{g}$ of the synthetic oestrogen diethylstilboestrol (DES) or vehicle only (olive oil; controls) for the first 5 days after birth. Embryos at the two-cell stage from control females were transferred to the oviducts of control females or DES-treated females (6-8-weeks-old). Immediately after transfer, the oviducts were removed and incubated in vitro for $6 \mathrm{~h}$. After incubation, slightly fewer embryos were recovered from oviducts exposed to DES compared with control oviducts (81\% versus $92 \% ; 0.05>P>0.01$ ). When the recovered embryos were cultured in vitro, $64 \%$ of the embryos from control oviducts reached the blastocyst stage in contrast to only $24 \%$ of those from oviducts of DES-treated mice; slightly fewer of the latter showed trophoblastic outgrowth $(76 \%$ versus $93 \% ; 0.05>P>0.01)$. Oviductal transport and uterine attachment was studied by introduction of blue-stained dextran microspheres into the oviduct. The microspheres appeared earlier in the uteri of DES-treated females than in the uteri of controls. Moreover, the spheres were not captured in the uterus in most DES-treated females, but they were trapped when a piece of Spongostan was placed in the uterine lumen. Scanning electron microscope studies showed differences between controls and DES-treated females in uterine mucosal lining of possible importance for embryo attachment to the surface epithelium. These studies showed that neonatal DES treatment caused changes in the adult reproductive tract as revealed by a detrimental effect upon embryo development, more rapid oviductal transport and loss of embryos from the uterus.
\end{abstract}

\section{Introduction}

Female mice treated with natural or synthetic oestrogens during the first few days after birth (oestrogenized females) are sterile in adult life (Takasugi and Bern, 1964; Forsberg et al., 1985). A major reason for this is a disturbed hypothalamic-pituitary control system resulting in acyclicity and anovulation (Plapinger and McEwen, 1978).

However, oestrogen-induced disturbances have been reported in other organs, incompatible with support of normal reproductive functions. After grafting ovaries from females exposed to diethylstilboestrol (DES) neonatally into ovariectomized control females, oocytes from the ovaries of DES-treated mice contributed to normal offspring; after superovulation, some of the females treated neonatally with DES mated but none became pregnant (Halling and Forsberg, 1990). When superovulation was combined with artificial insemination, most of the zygotes died at the two-cell stage and only a few examples of more advanced stages were recovered from the oviducts of DES-treated females (Halling and Forsberg, 1991). Zygote degeneration in females treated with oestrogen was also observed in experiments involving transfer of two-cell embryos from control females to the oviduct of other control females or

Received 6 July 1992. to females treated neonatally with DES (Halling and Forsberg, 1992).

In this study, the importance of local oviductal factors for zygote development was investigated under in vitro conditions. Oviductal transport activity was studied as well as the uterine capacity for embryo retention. Because the zygotes degenerate in the oviduct of oestrogenized females, dextran microspheres were used to study embryo transport.

\section{Materials and Methods}

\section{Animals}

The animals used were from a closed colony of the NMRI mouse strain, originally obtained from Charles River Wiga (Sulzfeld). They were kept in a controlled environment (temperature, humidity, $12 \mathrm{~h}$ light: $12 \mathrm{~h}$ dark), and fed a standard pellet diet (RIII; AB Ewos, Södertälje).

Females were allowed to give birth to their litters in separate cages. Within $24 \mathrm{~h}$ after birth, the sex of the newborn young was determined and every mother was allotted a pooled litter of six to eight females.

Females were treated s.c. with $5 \mu \mathrm{g}$ DES (Sigma Chemical Co., St Louis, MO) in $0.025 \mathrm{ml}$ olive oil, starting on the day of 
birth and for a further four times at $24 \mathrm{~h}$ intervals. Control females were treated according to an identical schedule with olive oil only.

\section{Recovery of embryos}

Embryos for transfer were obtained from 6-8-week-old control females superovulated with gonadotrophins and mated to normal males, as described by Halling and Forsberg (1991). On the second morning (08:00-10:00 h) after ovulation the females were killed by cervical dislocation and two-cell embryos were obtained by flushing the oviducts with M2 embryo culture medium (prepared according to Hogan et al., 1986) supplemented with $5 \mathrm{mg} \mathrm{BSA} \mathrm{ml}^{-1}$. Embryos from several females were pooled into $1 \mathrm{ml} \mathrm{M16}$ medium (Hogan et al., 1986) with $5 \mathrm{mg} \mathrm{BSA} \mathrm{m}^{-1}$ under paraffin oil. Embryos were kept on a heated stage $\left(37^{\circ} \mathrm{C}\right)$ when outside the incubator.

\section{Transfer of embryos and organ culture}

The two-cell embryos were transferred as soon as possible after recovery. The females were anaesthetized with Avertin $\left(0.02 \mathrm{ml} \mathrm{g}^{-1}\right.$ bodyweight of a $1.25 \%$ solution in saline of $10 \mathrm{~g}$ 2,2,2-tribromoethanol in $500 \mathrm{ml}$ 2-methylbutan-2-ol; Sigma/ BDH Chemicals Ltd). Ten embryos were transferred to the left oviduct of 8-week-old control females (irrespective of phase of the cycle) or DES-treated females. The technique for transfer was that described by Hogan et al. (1986). Immediately after transfer, the oviduct was carefully removed from the female without disturbing the position of the air bubbles that had been introduced with the embryos. The oviduct was placed on a piece of Millipore filter $(7 \mathrm{~mm} \times 7 \mathrm{~mm}$; pore size $0.45 \mu \mathrm{m})$ on top of a triangular metal grid, placed in a Falcon organ culture dish (Becton Dickinson Labware, Lincoln Park, NJ). The dish was filled with $1 \mathrm{ml}$ MEM (Flow Laboratories, Irvine) so that the filter just became wet. Incubation took place in an incubator at $37^{\circ} \mathrm{C}$ and $95 \%$ air $: 5 \% \mathrm{CO}_{2}$ for $6 \mathrm{~h}$.

\section{Embryo culture}

Media for embryo culture were prepared as described by Halling and Forsberg (1991).

After incubation, the oviducts were gently placed into a few drops of M2 medium and flushed with the same medium. Embryos were collected, counted and put into a 3037 Falcon organ culture dish containing I ml M16 medium with $5 \mathrm{mg}$ BSA ml ${ }^{-1}$. The embryos were cultured for 3 days and the number of blastocysts was then determined. The embryos were then put into organ culture dishes with DMEM containing $10 \%$ fetal calf serum (Flow Laboratories). After a further 3 days, the number of embryos that had hatched and that showed trophoblastic outgrowth on the plastic surface was determined.

\section{Oviductal transport and uterine retention of microspheres}

Six- to 8-week-old control and neonatally DES-treated females were superovulated with gonadotrophins (Halling and
Forsberg, 1991). On the morning after ovulation (08:0010:00 h), female mice were anaesthetized with Avertin as described above and six blue-stained dextran microspheres (DMB-5, Pharmacia, Uppsala) were introduced into the left oviductal ampulla using a technique described by Hogan et al. (1986), except that no air bubbles were introduced with the spheres. To ensure that the size of the microspheres installed was as closely similar to the size of two-cell embryos as possible, a sample of microspheres was suspended in culture medium before installation and those microspheres similar in size to two-cell embryos were picked out under a stereomicroscope (SMZ-10, Nikon, Tokyo) and used for the experiments.

At different times $(24,36,48$ and $72 \mathrm{~h})$ after transfer, the left ovary, oviduct and uterus were removed en bloc and put into a $35 \mathrm{~mm}$ Petri dish (Nunc, Denmark) with $0.5 \mathrm{ml}$ phosphatebuffered saline (PBS), pH 7.0. The blue-stained microspheres were observed and localized through the oviductal and uterine walls in the stereomicroscope, with both episcopic and diascopic illumination. The number of microspheres in the oviduct and uterus were counted separately.

In one experiment, control females were injected with $\mathrm{I} \mu \mathrm{g}$ oestradiol in PBS-ethanol (5\% ethanol) immediately after installation of microspheres and again $24 \mathrm{~h}$ later (oestrogen dominated females). The females were killed $48 \mathrm{~h}$ after installation and the microspheres counted as described above.

In initial experiments, many of the microspheres were lost from the uterus and oviduct in DES-treated females $48 \mathrm{~h}$ after transfer. To verify that this was due to a hastened oviductal transport or a possible reduced uterine retention, a piece ( $1 \mathrm{~mm} \times 3 \mathrm{~mm}$ ) of sterile Spongostan (Ferrosan, Copenhagen) was inserted immediately after transfer of the microspheres through a small longitudinal incision, between the upper and the middle third of the left uterine horn. The small uterine wound was closed by a single suture. When the females were killed $48 \mathrm{~h}$ later, the uterine horn did not show dilatation or hyperaemia as signs of irritation compared with the contralateral horn.

\section{Scanning electron microscopy}

Eight-week-old control and neonatally DES-treated females were superovulated as described above. On the morning after ovulation, five control and five DES-treated females were killed. Specimens from both uterine horns were fixed in $2.5 \%$ glutaraldehyde in $0.1 \mathrm{~mol}$ phosphate buffer $\mathrm{l}^{-1}(\mathrm{pH} 7.3)$ for $2 \mathrm{~h}$, postfixed in $1 \%$ osmium tetroxide for $\mathrm{Ih}$, washed in buffer and dehydrated in a graded series of ethanol. Finally, the preparations were dried in a Balzers 030 Critical Point Dryer and sputter-coated with gold-palladium. The specimens were examined in a Philips 515 scanning electron microscope.

Photographs were taken at ten random locations on specimens from each female at magnifications of $\times 163, \times 1310$ and $\times 5200$.

\section{Statistical analysis}

Differences in incidences between different groups were compared using the $\chi^{2}$ test with Yates correction for continuity (Zar, 1984). 


\section{Results}

\section{Studies of embryo development after incubation in isolated oviducts}

The experiments were repeated on three different occasions without any statistical differences in results between the three occasions. The results from the different occasions were therefore pooled.

The total material consisted of 12 oviducts (120 transferred embryos) from 12 control females and 11 oviducts (110 transferred embryos) from II DES-treated females. After incubation, all of the recovered embryos were still at the two-cell stage. Fewer embryos were recovered from oviducts of DEStreated mice than from control oviducts $(80.9 \%$ versus $91.7 \%$; $0.05>P>0.01)$. Seventy out of 110 embryos $(63.6 \%)$ recovered from oviducts of control animals developed into blastocysts after incubation in vitro; whereas 21 of 89 embryos $(23.6 \%)$ were recovered from oviducts of control animals. This difference was significant $(0.01>P>0.001)$. Finally, 65 out of 70 blastocysts $(92.9 \%)$ emanating from oviducts of control animals and 16 of $21(76.2 \%)$ from oviducts of DES-treated mice developed to the implantation stage $(0.05>P>0.01)$.

\section{Oviductal transport and uterine retention of microspheres}

Six blue-stained microspheres of about the same size as the ova were installed into the left oviduct of female mice neonatally treated with DES and control animals to determine whether there were differences in oviductal transport between control and DES-treated females.

When control females were killed $24 \mathrm{~h}$ after installation of the microspheres, all of the six microspheres installed were recovered from the oviduct of each of 13 control females (Fig. 1). A similar result was obtained for six of 12 DES-treated females killed at the same time. In the remaining DES-treated females, some microspheres were lost and in one female none was recovered. In three females, one or two microspheres were found in the uterus.

Two days after introduction of microspheres, they were all recovered from 10 of 14 oviducts of control animals. In the remaining cases, some microspheres were lost and those recovered were found in the oviduct or uterus. In contrast, no microspheres were recovered from seven of 12 DES-treated females, one to six spheres were found in the oviducts of four females and, in a final case, a single microsphere was found in the uterus.

Three days after insertion, two to six microspheres were recovered from the oviduct of 12 out of 13 control females studied; in five of these females, some microspheres were present in the oviduct. In a single female, all six spheres were still in the oviduct. Among 14 DES-treated females, no microspheres could be found in six females; in seven females two to four microspheres were localized in the oviduct and in the other female only two were recovered from the uterus.

The situation was quite different in the 13 DES-treated females in which oviductal installation of microspheres was combined with Spongostan insertion into the uterus (Fig. 2a). All six microspheres were recovered from $100 \%$ of these DEStreated females, but with a different interindividual distribution. (a)

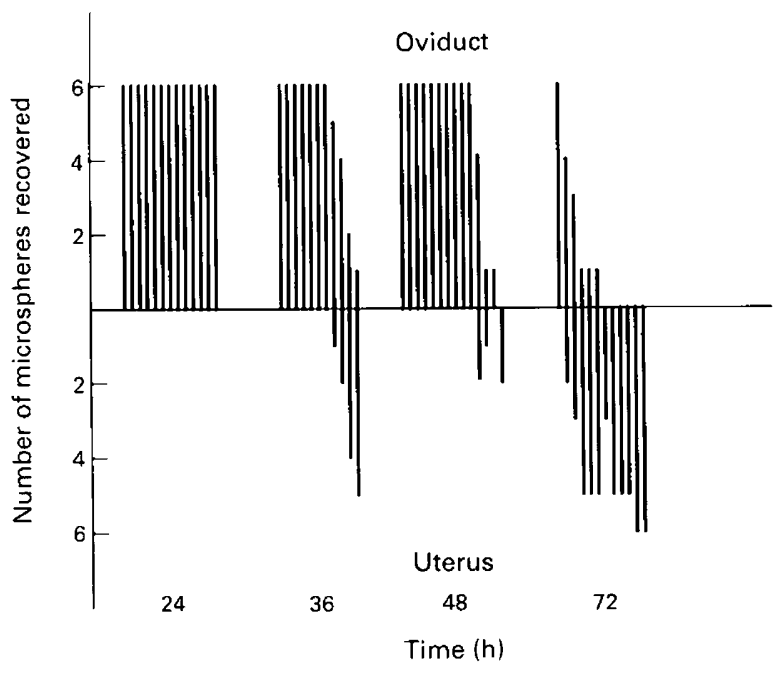

(b)

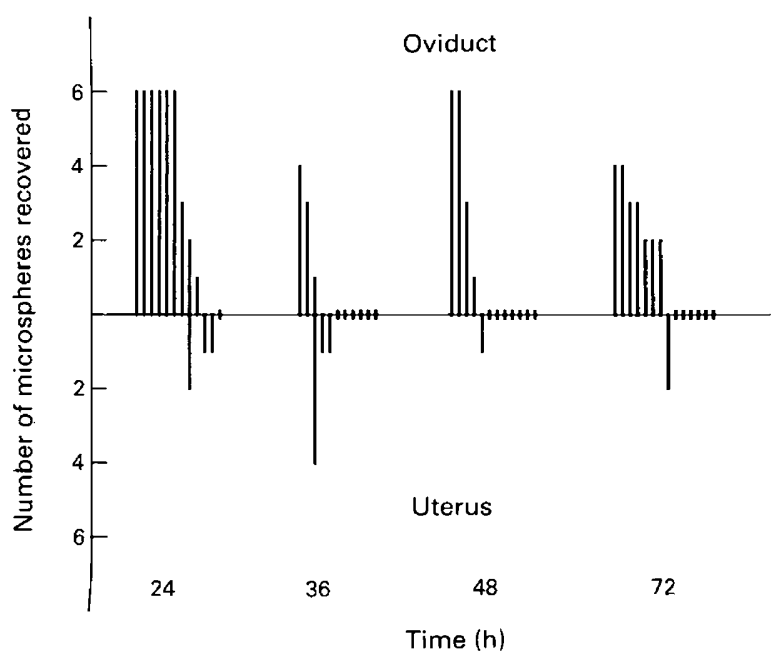

Fig. 1. Schematic illustration of results from studies on oviductal and uterine transport activity of microspheres. Six microspheres were introduced through the oviductal ampulla and their localization in the oviduct and uterus were studied $24,36,48$ and $72 \mathrm{~h}$ later in (a) control and (b) DES-treated females. The number of microspheres recovered from both regions (oviduct; uterus) in individual females (represented by vertical lines) is given along the vertical axis.

In two females all six microspheres were localized in the oviduct; in five cases they were all trapped in the Spongostan. In the remaining six females the localization of microspheres was both oviductal and uterine in the Spongostan, with a varying individual proportion of microspheres in the two regions.

Oestradiol treatment of 12 females that had not been treated previously (oestradiol dominated females) resulted in a similar distribution of microspheres as seen in DES-treated females with Spongostan in the uterus (Fig. 2b). Microspheres were recovered from the oviduct or uterus in all cases. In two cases all microspheres were retrieved in the oviduct and in a further 
(a)

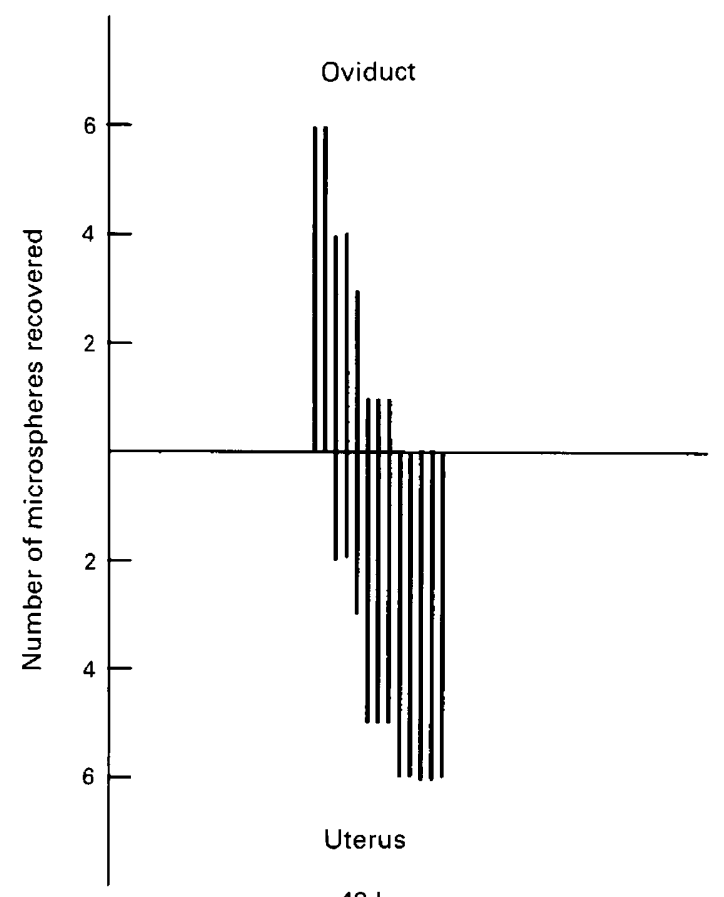

$48 \mathrm{~h}$

(b)

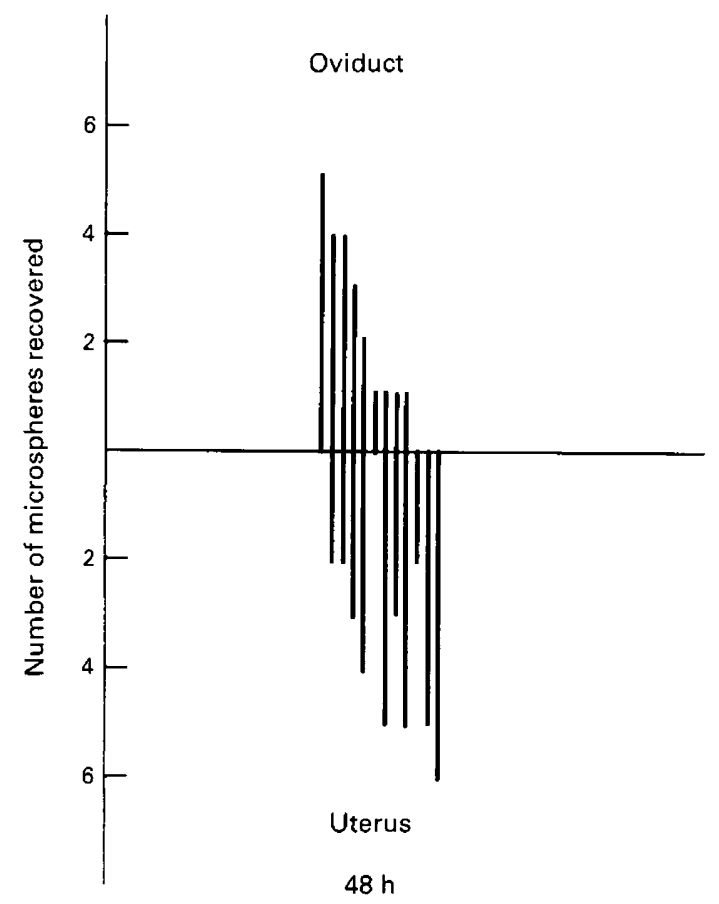

Fig. 2. Schematic illustrations of the results from studies on microsphere distribution between the oviduct and uterus in (a) females treated with diethylstilboestrol (DES) and (b) in females not treated with DES but treated with oestradiol (oestradiol dominated females) after insertion of a piece of Spongostan into the uterus. The number of microspheres recovered from the oviduct and uterus in individual females (represented by vertical lines) is given along the vertical axis. three cases only in the uterus. The total number of microspheres recovered varied from one (one female) to six (seven females).

\section{Scanning electron microscope studies}

In control females, the uterine mucosa was arranged into a regular pattern of ridges, perpendicular to the longitudinal axis of the uterine horn, and separated by clefts (Fig. 3a). These ridges had a substructure with tightly packed, slender cells arranged into stacked 'slices' separated by deep grooves (Fig. 3c).

In DES-treated females the mucosal surface was irregularly undulating with low peaks separated by shallow grooves (Fig. 3b); the epithelial cells were distended in their apical part and arranged in an irregular pattern (Fig. 3d). The cells of control uteri were largely covered with club-like microvilli or a meshwork of thread-like microvilli (Fig. 3e). In uteri of DEStreated mice, only the latter type of microvilli was seen (Fig. 3f). These anomalies were observed in all preparations from DES-treated females.

\section{Discussion}

The experiments reported here show that neonatal exposure of female mice to DES caused changes in the function of their reproductive tract later in life, which may have contributed to their infertility. Even brief exposure to the oviduct of such animals reduced the potential of control two-cell embryos to develop to blastocysts, suggesting the presence of harmful factors in the oviductal milieu. The transport of microspheres through the oviduct was accelerated and would have exposed embryos to an inappropriate uterine environment if similar effects occur with embryos. The retention of spheres within the uterus was also reduced.

The observed effects of the oviduct milieu confirm and extend earlier observations (Halling and Forsberg, 1990, 1991, 1992). In the study reported here, we have demonstrated that even a brief $(6 \mathrm{~h})$ in vitro exposure of two-cell embryos from control females to the luminal environment of oviducts from DES-treated females is detrimental for later normal zygote development. The injurious oviductal influence was apparent as slightly fewer two-cell embryos were recovered from oviducts of DES-treated mice than from oviducts of control animals after the incubation period, but the most pronounced effect was the reduced blastocyst formation when recovered embryos were further cultured in vitro. Finally, the incidence of blastocysts reaching the implantation stage (trophoblastic outgrowth) was slightly reduced after embryo passage in oviducts of DEStreated mice. The results support the conclusion that functional disturbances in the female genital tract of mice prenatally (CD-1) or neonatally (NMRI) exposed to DES result in reduced preblastocyst developmental potential (Wordinger et al., 1989).

The concentration of total plasma oestrogen was not different in 8-month-old female mice exposed to DES on days 9-16 of gestation compared with females treated with vehicle only (McLachlan et al., 1982). However, attempts to determine plasma oestrogen in mice using radioimmunoassay are severely hampered, because of a factor crossreacting with antiserum to 

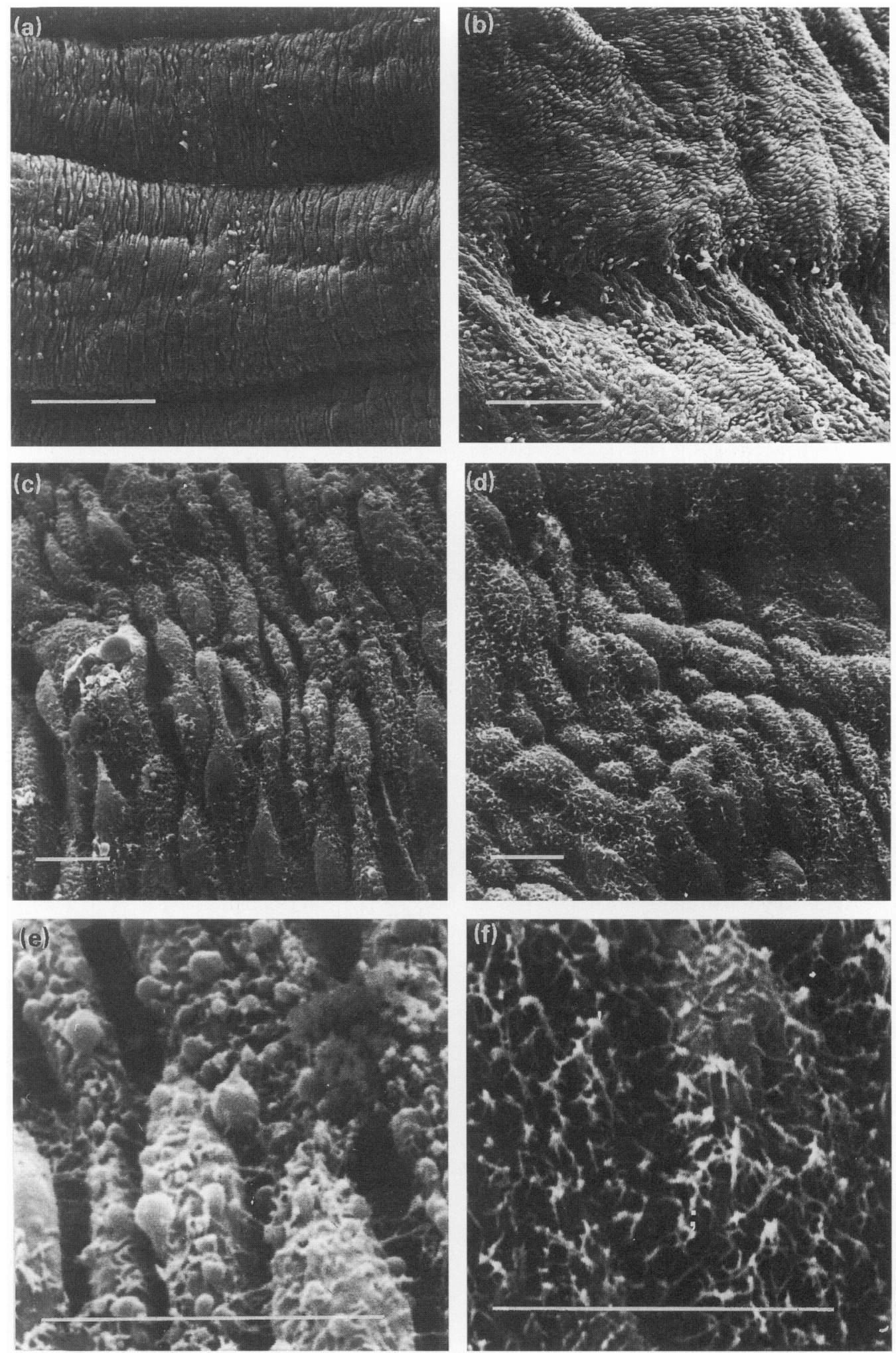

Fig. 3. Representative photographs from scanning electron microscope (SEM) studies. (a) The uterine mucosal surface of control females formed a regular pattern of ridges and clefts; ibi in diethylstilboestrol (DES)-treated females an irregular pattern with shallow mucosal folds was seen. (c) The ridges in control uteri were formed by stacked 'slices', consisting of tightly packed slender cells. (d) The cells in uteri of DES-treated mice were distended in their apical part. (e) The cells of control uteri were covered with club-like and thread-like microvilli. (f) In uteri of DES-treated mice only the latter type of microvilli was seen. Scale hars (a) (b) $0.1 \mathrm{~mm}$; (c)-(f) $10 \mu \mathrm{m}$. 
oestradiol (Barkley et al., 1985; J-G. Forsberg, unpublished observations). Thus, we do not know the actual concentration of circulating oestrogen. Because early embryos can survive in vitro in a medium containing far higher oestradiol concentrations than occur in plasma, the possible oestradiol effect may not be directly on the embryo (Cline et al., 1977). When embryos were cultured in oviductal fluid from oestrogen dominated females or transferred to the oviduct of such females, the viability was markedly reduced (Cline et al., 1977; Halling and Forsberg, 1992). Thus a detrimental effect of oestrogen on embryo development could include a reduced amount of a required factor or the presence of an oestrogen-dependent inhibitory factor.

Ovum and zygote transport through the oviduct depends on rigidity of the oviductal wall, activity of ciliated and secretory cells, and viscosity of luminal secretions, factors that are under hormonal control (Jansen, 1984). Oestrogen accelerates embryo transport through the isthmic part of the rat oviduct (Forcelledo et al., 1986; Moore and Croxatto, 1988).

We used stained microspheres of the same size as two-cell embryos to mimic embryo transport through the oviduct and uterus. Even though there are reasons to believe that there could be differences in transport mechanisms between the inert surrogate material and the living embryo (Jansen, 1984), studies in rats indicate that transport of starch microspheres is similar to transport of native eggs (Moore and Croxatto, 1988).

Microspheres were recovered from the uterus of DES-treated females at $24 \mathrm{~h}$ and from control uterus at $36 \mathrm{~h}$ after introduction into the oviduct, which points to a more rapid transport in the oviducts of DES-treated mice compared with control animals. The most striking difference between DES-treated and control females at the later stages studied $(36,48$ and $72 \mathrm{~h}$ ) was that more microspheres were lost in DES-treated females than in control females. No microspheres were recovered in about half of the DES-treated females studied. Because $100 \%$ of the spheres were recovered from the uterus from DES-treated females with a Spongostan implant, this material obviously functioned as a trap, preventing the microspheres from being rapidly expelled through the uterus and vagina. The oestradiol dominated females that had not been treated with DES had a distribution pattern of microspheres between the oviduct and uterus that was similar to that in DES-treated females with Spongostan in the uterus. Oestradiol treatment accelerated the transport of microspheres from the oviduct into the uterus but, in contrast to the situation in DES-treated females without Spongostan, the microspheres were not lost from the uterus. Thus, there must be a functional disturbance in the uterus of DES-treated females that does not allow the spheres to be retained as in controls.

In the rat uterus, but not in the oviduct, a differential transport of fertilized and unfertilized ova and expulsion of the latter has been described by Villalón et al. (1982). This could be explained by local hormonal factors related to the embryo or physicochemical characteristics of the embryonic zona pellucida affecting contacts with the uterine epithelial surface (Villalón et al., 1982). Trophoblast contact with the uterine epithelium influences differentiation of the latter and oil can mimic this reaction (Pollard and Finn, 1974). In rats, the uterine luminal membrane attains a special appearance at the time of blastocyst attachment (Nilsson, 1966).
Oviducts from females exposed to DES prenatally or neonatally are morphologically different from control oviducts. The oviducts of DES-treated mice have an abnormal mucosal folding; the coiling pattern is simple; and the intrabursal length is reduced (Newbold et al., 1983; Wordinger and Morrill, 1985; Halling and Forsberg, 1992). These anomalies may influence oviductal motility and embryo transport. A disorganization of the uterine muscle compartment was described by Ostrander et al. (1985). In the study reported here, the uterine mucosal surface of DES-treated females was different from that in control females with respect to cellular arrangement, apical cell structure and morphology of the microvilli. As a consequence, the uterine mucosa may not be able to retain the embryos, as occurs normally in the earliest apposition phase of implantation (Enders and Schlafke, 1967). Maternal and embryonic cells are then in close contact; the uterine luminal cell membrane has numerous villi and ruffles where it is related to the blastocyst (Potts, 1966; Hedlund and Nilsson, 1971). The difference in uterine mucosal surface between DES-treated females and control females might be linked to functional differences in embryo attachment and it could explain embryo loss in DES-treated females.

This investigation was supported by grants from the Swedish Medical Research Council (project no. B92-12X-09056-03A), the Crafoord Foundation, Lund, Sweden and from the Swedish Society for Medical Research, Department of Pharmacology, Karolinska Institute, Stockholm, Sweden.

\section{References}

Barkley MS, Lasley BL, Thompson MA and Shackleton CL (1985) Equol: a contributor to enigmatic immunoassay measurements of estrogen Steroids $\mathbf{4 6}$ $587-608$

Cline MC, Randall PA and Oliphant G (1977) Hormone-mediated oviductal influence on mouse embryo development Fertility and Sterility 28 766-771

Enders AC and Schlafke S (1967) A morphological analysis of the early implantation stages in the rat American Joumal of Anatomy 120 185-226

Forcelledo ML, de la Cerda ML and Croxatto HB (1986) Effectiveness of different estrogen pulses in plasma for accelerating ovum transport and their relation to estradiol levels in the rat oviduct Endocrinology 119 1189-1194

Forsberg J-G, Tenenbaum A, Rydberg C and Sernvi C (1985) Ovarian structure and function in neonatally estrogen treated female mice. In Estrogens in the Environment Vol. II, pp 327-346 Ed. JA McLachlan. Elsevier Science Publishers, New York

Halling A and Forsberg J-G (1990) Ovarian reproductive function after exposure to diethylstilbestrol in neonatal life Biology of Reproduction 43 472-477

Halling A and Forsberg J-G (1991) Effects of neonatal exposure to diethylstilbestrol on early mouse embryo development in vivo and in vitro Biology of Reproduction 45 157-162

Halling A and Forsberg J-G (1992) The functional importance of the oviduct in neonatally estrogenized mouse females for early embryo survival Teratology $4575-82$

Hediund $\mathrm{K}$ and Nilsson $\mathrm{O}$ (1971) Hormonal requirement for the uterine attachment reaction and blastocyst implantation in the mouse, hamster and guinea-pig Journal of Reproduction and Fertility 26 267-269

Hogan B, Constantini F and Lacy E (1986) Manipulating the Mouse Embryo: $A$ Laboratory Manual Cold Spring Harbor, New York

Jansen RPS (1984) Endocrine response in the Fallopian tube Endocrine Reviews 5 525-551

McLachlan JA, Newbold RR, Shah HC, Hogan MD and Dixon RL (1982) Reduced fertility in female mice exposed transplacentally to diethylstilbestrol (DES) Fertility and Sterility 38 364-371

Moore GD and Croxatto HB (1988) Effects of delayed transfer and treatment with oestrogen on the transport of microspheres by the rat oviduct Journal of Reproduction and Fertility 83 795-802 
Newbold RR, Bullock BC and McLachlan JA (1983) Exposure to diethylstilbestrol during pregnancy permanently alters the ovary and oviduct Biology of Reproduction 28 735-744

Nilsson $O$ (1966) Structural differentiation of luminal membrane in rat uterus during normal and experimental implantations Zeitschrift für Anatomie und Entwicklungsgeschichte 125 152-159

Ostrander PL, Mills KT and Bern HA (1985) Long-term responses of the mouse uterus to neonatal diethylstilbestrol treatment and to later sex hormone exposure Joumal of the National Cancer Institute 74 121-135

Plapinger L and McEwen BS (1978) Gonadal steroid-brain interactions in sexual differentiation. In Biological Determinants of Sexual Behaviour, pp 153-218 Ed. JB Hutchinson. John Wiley, New York

Pollard RM and Finn CA (1974) Influence of the trophoblast upon differentiation of the uterine epithelium during implantation in the mouse Joumal of Endocrinology 62 669-674

Potts M (1966) The attachment phase of ovoimplantation American Journal of Obstetrics and Gynecology 96 1122-1128
Takasugi N and Bern HA (1964) Tissue changes in mice with persistent vaginal cornification induced by early postnatal treatment with estrogen Joumal of the National Cancer Institute 33 855-865

Villalón M, Ortiz ME, Aguayo C, Munoz J and Croxatto HB (1982) Differential transport of fertilized and unfertilized ova in the rat Biology of Reproduction 26 337-341

Wordinger RJ and Morrill A (1985) Histology of the adult mouse oviduct and endometrium following a single prenatal exposure to diethylstilbestrol Virchows Archio [Cell Pathology] 50 71-79

Wordinger RJ, Brown D, Atkins E and Jackson FL (1989) Superovulation and early embryo development in the adult mouse after prenatal exposure to diethylstilboestrol Joumal of Reproduction and Fertility 85 383-388

Zar JH (1984) Biostatistical Analysis. (2nd edn) Prentice-Hall Inc., Englewood Cliffs 